\title{
Study on the Time-Scale Similarity of Bouc-Wen Hysteresis Model
}

\author{
Ning Dong ${ }^{1, a}, J_{i a} G^{1, b}$, Xiangdong Liu ${ }^{1, c}$ and Xuefei Mao ${ }^{1, d}$ \\ ${ }^{1}$ School of automation, Beijing Institute of Technology, Beijing 100081, China. \\ adongning@bit.edu.cn, bgaojia2009.www@163.com, 'xdliu@bit.edu.cn, dmaoxuefei21@163.com
}

Keywords: Bouc-Wen model, hysteresis, time-scale similarity.

\begin{abstract}
This paper will focus on Bouc-Wen hysteresis model to study the time-scale similarity of the hysteresis. Considering different hysteresis characteristics of Bouc-Wen model, this paper studies the time-scale similarity between the output curves of different hysteresis characteristics under the excitation of the triangular waves with different frequencies, and the Euclidean distance function is used to measure the curve similarity. The time-scale similarity proposed in this paper will contribute to establishing a dynamic similarity model, which is without complex operation and don't need a lot of storage space.
\end{abstract}

\section{Introduction}

As a nonlinear analytical form of the hysteresis loop, Bouc-Wen model is widely used in the modeling of hysteresis and can describe a lot of hysteresis phenomena existing in various engineering fields such as mechanical, civil, and materials. Therefore, Bouc-Wen hysteresis model has attracted many researchers' attention and various modeling methods have been proposed in the past decades[1-4].

Besides, several approaches for modeling the hysteresis based on empirical observations have been developed[5-7]. But these empirical models are not rate-dependent and the memory-based hysteresis model still needed many memory units. Thus, literature [8] has taken the rate-dependence and fewer memory units into account and proposed a rate-dependent empirical model without using many memory units based on the geometric similarity which describes the similarity among the output curves in static and the time-scale similarity which discloses the relationship of the output curves with different frequencies.

For Bouc-Wen model, we have studied the geometric similarity before, which describes the similarity among the output curves with different extremes. However, as the hysteresis of Bouc-Wen model is rate-dependent, in order to fully describe the hysteresis, the frequency must be considered. Thus, based on Bouc-Wen hysteresis model, this paper presents the time-scale similarity and the Euclidean distance function is used to measure the curve similarity of different hysteresis characteristics.

\section{The mathematical description and hysteresis characteristics of classical Bouc-Wen model}

Classical Bouc-Wen model can be described as:

$$
\dot{z}=\alpha d \cdot \dot{u}-\beta|\dot{u}||z|^{n-1} z-\gamma \dot{u}|z|^{n}
$$

$u$ is the input signal; $z$ is the hysteresis output; the parameter $\alpha$ determines the amplitude size of the hysteretic restoring force. $\beta$ and $\gamma$ determine the shape of the hysteresis curve. $d$ is a constant. In the elastic structure and material, $n$ usually takes 1 , and then (1) can be simplified as:

$$
\dot{z}=\alpha d \cdot \dot{u}-\beta|\dot{u}| z-\gamma \dot{u}|z|
$$

As described by (2), through the proper selection of $\alpha, \beta$, $\gamma$, different hysteresis curves can be obtained. In general, $\alpha$ and $\beta$ are positive, and $\gamma$ can be positive or negative[9,10]. Taking $d=0.116, \alpha$ $=1.0$, for $u=\sin (2 \pi t)$, with different values of $\beta$ and $\gamma$, the hysteresis output $z$ shows hard (a) and soft (b) characteristics, as shown in figure 1. 


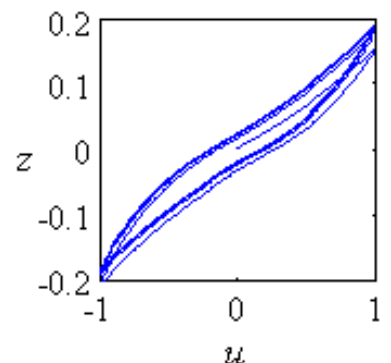

(a) $\beta=1, \gamma=-1$

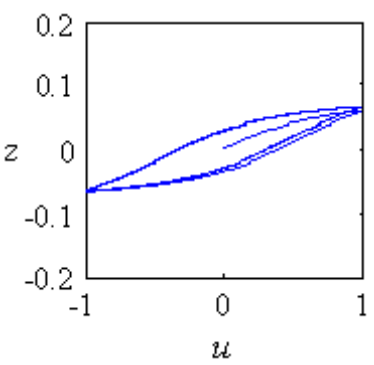

(b) $\beta=1, \gamma=1$

Fig. 1 The hysteresis curve of different parameter combinations

\section{The time-scale similarity of Bouc-Wen hysteresis model}

The time-scale similarity phenomena. Firstly, two sets of triangular signals whose only difference is the frequency will be used here, as shown in figure 2. The different frequencies are labeled as $f_{0}$ and $f\left(f_{0}<f\right)$, and the cycle are $T_{0}$ and $T$. The output curves are shown in figure 3 . And use $D_{T_{0}}^{0}(n)$ and $D_{T}(n)$ to describe the two output curves.

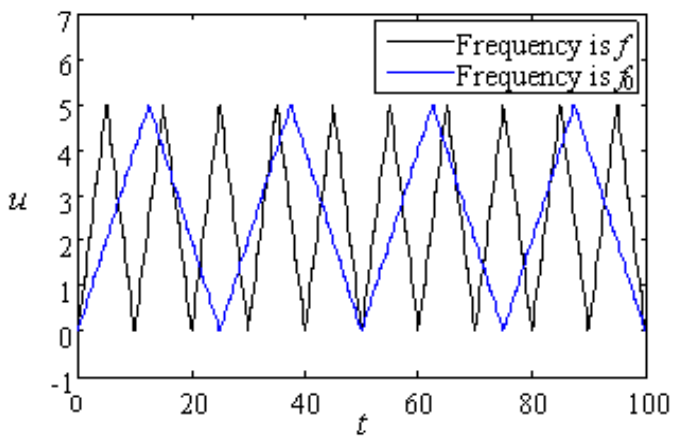

Fig. 2 Two sets of triangular signals with different frequencies

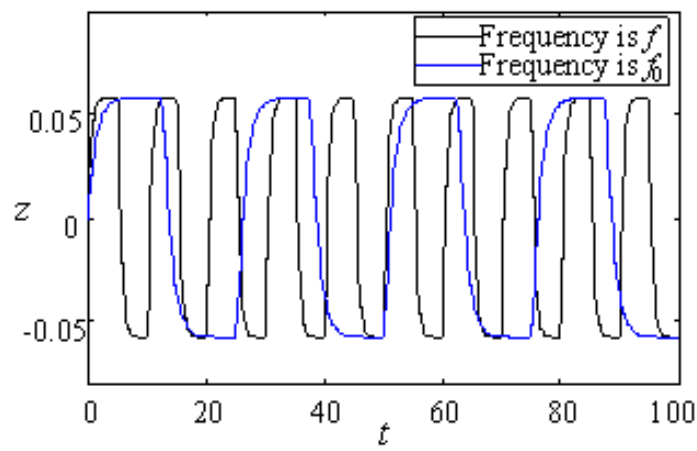

(a)

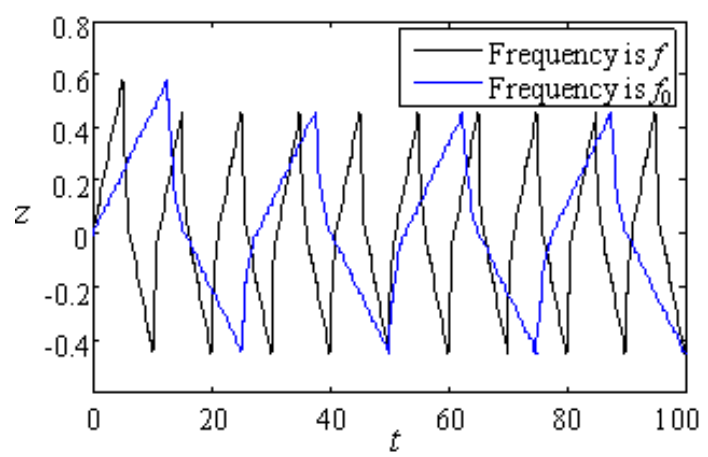

(b)

Fig. 3 Output curves of (a) soft characteristic and (b) hard characteristic

Similarity measure. In order to compare two output curves, the time-scale amplification needs to be carried out. If the cycle of $D_{T_{0}}^{0}(n)$ is amplified $N_{T}\left(N_{T}=f_{0} / f<1\right)$ times to make $N_{T} \cdot\left(1 / f_{0}\right)=1 / f$ as in (3), then the curves after time-scale amplification (labeled $D_{T_{0}}(n)$ ) has the same cycle with $D_{T}(n)$. The time-scale amplification of $D_{T_{0}}^{0}(n)$ to $D_{T_{0}}(n)$ is shown in figure 4(a) (soft characteristic) and 4(b) (hard characteristic).

$$
D_{T_{0}}(n)=\Gamma_{\text {time-scale }}\left(D_{T_{0}}^{0}(n)\right)
$$

As can be seen from figure 4, the two curves are similar but not coincident. So the similarity between $D_{T_{0}}(n)$ and $D_{T}(n)$ is measured by Euclidean distance as follows.

$$
\operatorname{simf}\left(D_{T_{0}}(n), D_{T}(n)\right)=\left[\sum_{i=1}^{n}\left(D_{T_{0}}(n)-D_{T}(n)\right)^{2}\right]^{\frac{1}{2}}
$$




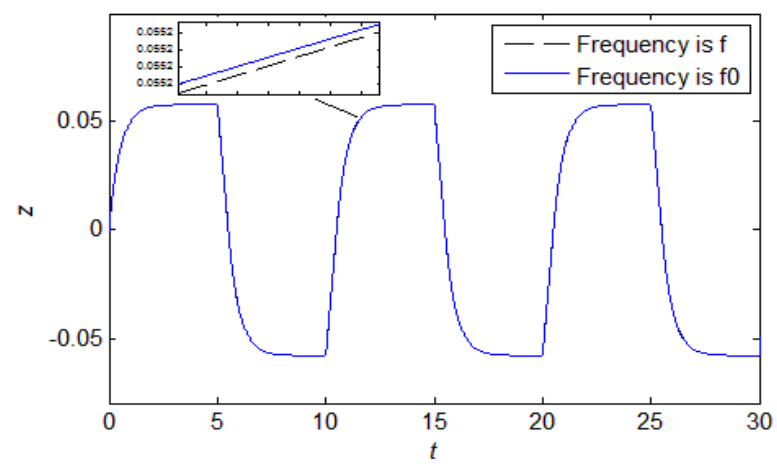

(a)

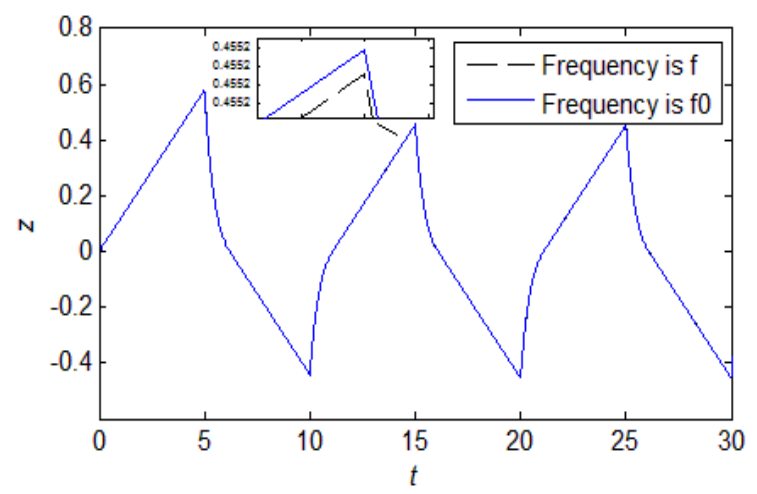

(b)

Fig. 4 Curves after time-scale amplification of (a) soft characteristic and (b) hard characteristic

The similarity between the output curve $D_{T_{0}}(n)$ which is the time-scale amplification of $D_{T_{0}}^{0}(n)$ whose frequency is $0.001 \mathrm{~Hz}$ and $D_{T}(n)$ whose frequency is $0.01 \mathrm{~Hz}, 0.1 \mathrm{~Hz}, 1 \mathrm{~Hz}, 10 \mathrm{~Hz}$ are measured by amplifying the curve $D_{T_{0}}(n)$ by some times and move it by some phases as shown in table 1 (soft characteristic) and 2 (hard characteristic).

Table 1 The similarity and error between $D_{T_{0}}(n)$ and $D_{T}(n)$ of soft characteristic

\begin{tabular}{|c|c|c|c|c|}
\hline Frequency & $0.01 \mathrm{~Hz}$ & $0.1 \mathrm{~Hz}$ & $1 \mathrm{~Hz}$ & $10 \mathrm{~Hz}$ \\
\hline Euclidean distance & $3.5238 \mathrm{e}-06$ & $4.7945 \mathrm{e}-05$ & 0.0032 & 0.0157 \\
\hline Amplification & 1.0000 & 1.0000 & 0.9983 & 0.9915 \\
\hline Phase & 0 & 0 & 0 & 0 \\
\hline Error range & $\begin{array}{c}-1.5 e-06 \sim+1.5 e- \\
06\end{array}$ & $-2 \mathrm{e}-05 \sim+2 \mathrm{e}-05$ & $-0.0002 \sim 0.0002$ & $-0.0006 \sim+0.0006$ \\
\hline Mean square error & $1.1077 \mathrm{e}-07$ & $1.5073 e-06$ & $2.1756 \mathrm{e}-05$ & 8.8249e-05 \\
\hline
\end{tabular}

Table 2 The similarity and error between $D_{T_{0}}(n)$ and $D_{T}(n)$ of hard characteristic

\begin{tabular}{|c|c|c|c|c|}
\hline Frequency & $0.01 \mathrm{~Hz}$ & $0.1 \mathrm{~Hz}$ & $1 \mathrm{~Hz}$ & $10 \mathrm{~Hz}$ \\
\hline Euclidean distance & 2.6425e-06 & $1.3116 \mathrm{e}-05$ & 1.5269e-04 & $2.2620 \mathrm{e}-04$ \\
\hline Amplification & 1.0000 & 1.0000 & 1.0000 & 1.0000 \\
\hline Phase & 0 & 0 & 0 & 0 \\
\hline Error range & $-2 e-06 \sim+2 e-06$ & $\begin{array}{c}-7.5 e-06 \sim+7.5 e- \\
06\end{array}$ & $\begin{array}{c}-2.5 e-05 \sim+2.5 e- \\
05\end{array}$ & $\begin{array}{c}-0.00015 \sim+0.000 \\
15\end{array}$ \\
\hline Mean square error & $8.1886 \mathrm{e}-08$ & $4.0642 \mathrm{e}-07$ & 3.4782e-06 & $6.2818 \mathrm{e}-06$ \\
\hline
\end{tabular}

As shown in table 1 and 2, when the hysteresis shows soft (hard) characteristic, the similarity measure value between $D_{T_{0}}(n)$ and $D_{T}(n)$ are respectively 3.5238e-06, 4.7945e-05, 0.0032, 0.0157 (2.6425e-06, 1.3116e-05, 1.5269e-04, 2.2620e-04), which indicates that $D_{T_{0}}(n)$ and $D_{T}(n)$ have high similarity. In fact, as the errors are within the scope of $-0.0006 \sim 0.0006(-0.0002 \sim 0.0002)$ and the mean square errors are not greater than 0.0001(0.00001), $D_{T_{0}}(n)$ and $D_{T}(n)$ are almost equal. We can also conclude that with the increase of the frequency difference, the similarity measure value and the error become larger, which indicates that the similarity degree is lower with the larger frequency difference.

\section{Summary}

In this paper, the time-scale similarity of the Bouc-Wen model has been presented. Firstly, the time-scale amplification has been carried out to compare the two output curves. Then the Euclidean distance function is used to measure the similarity between the two curves, which indicates that the time-scale similarity degree is extremely high and with the frequency difference increasing, the similarity degree is becoming lower. On the whole, the time-scale similarity degree is so high that the two output curves are almost coincident. 


\section{Acknowledgments}

This study was supported by Beijing Natural Science Foundation of China (Grant No.4122066), National Natural Science Foundation of China (Grant No.11572035) and Beijing Institute of Technology Research Fund Program for Young Scholars.

\section{Corresponding author}

Xuefei Mao, School of automation, Beijing Institute of Technology, 5 South Zhongguancun Street, Haidian District, Beijing 100081, China.

Email: maoxuefei21@163.com.

\section{References}

[1] I.D. Mayergoyz. Mathematical models of hysteresis. Berlin, Germany: Springer-Verlag (1991)

[2] Zeng Silin, Dang Xuanju, Zhang Lin. Improvement for Bouc-Wen hysteresis model and description on non-smooth hysteresis characteristic of voice coil motor. Journal of Vibration and Shock. 29 (2010) 82-85,112.

[3] Luca Facchini, Michele Betti. An efficient Bouc \& Wen approach for seismic analysis of masonry tower[J]. Frattura e Integrita Strutturale. 29 (2014) 139-149.

[4] Fayçal Ikhouanea; Víctor Mañosaa and José Rodellara. Dynamic properties of the hysteretic Bouc-Wen model. Systems and Control Letters. Vol. 56 (2007) No. 3, p. 197-205.

[5] Saeid Bashash, Nader Jalili. Underlying memory-dominant nature of hysteresis in piezoelectric materials. Journal of Applied Physics. Vol. 100 (2006) No. 1, p. $014103-014109$.

[6] Saeid Bashash, Nader Jalili, Phillip Evans, Marcelo J. Dapino. Recursive memory-based hysteresis modeling for solid-state smart actuators. Journal of Intelligent Material Systems and Structures. Vol. 20 (2009) No. 18, p. 2161-2171.

[7] Saeid Bashash, Nader Jalili. A polynomial-based linear mapping strategy for feed forward compensation of hysteresis in piezoelectric actuators. Journal of Dynamic Systems, Measurement and Control. Vol. 130 (2008) No. 3, p. 1-10.

[8] Zhi-Lin Lai, Zhen Chen, Xiang-Dong Liu, Qing-He Wu. A Novel Similarity-Based Hysteresis Empirical Model for Piezoceramic Actuators Sensors \& Actuators: A. Physical 2013, 197C: 150-165.

[9] Li Hongguang, He Xu, Meng Guang. Numerical Simulation for Dynamic Characteristics of Bouc-Wen Hysteretic System. Journal of System Simulation. Vol. 16 (2004) No. 9, p. 2009-2011, 2036.

[10]Yan Zhihao. Research on parameter identification of nonlinear structure based on the Bouc-Wen model. Nanjing University of Science and Technology (2005) 